\title{
Virtual-EMI Lab: Removing Mysteries From Black-magic To A Successful Front-End Design *
}

\author{
Hany Fahmy ${ }^{\# 1}$, Chen Wang ${ }^{\# 2}$, Davy Pissoort ${ }^{*}$, and Amolak Badesha ${ }^{\dagger}$ \\ ${ }^{\#}$ Nvidia Corporation \\ 2701 San Tomas Expressway, Santa Clara, CA, 95050, USA. \\ ${ }^{1}$ chenwangenvidia.com \\ ${ }^{2}$ hfahmy@nvidia.com \\ *FMEC-KHBO, Dept. IW\&T \\ Zeedijk 101, Oostende, Belgium \\ davy.pissoort@khbo.be \\ ${ }^{\dagger}$ Agilent Technologies \\ 5301 Stevens Creek Blvd, Santa Clara, CA 95051, USA. \\ amolak_badesha@agilent.com
}

\begin{abstract}
EMI engineers are struggling everyday with complex radiation problems that fail critical products to pass EMI certification and causes big loss of profit. Advances in EMI engineering are following a similar trend like Signal-Integrity engineering 10-years ago when simulation tools became capable of providing accurate predictive simulations in a reasonable amount of time. With careful engineering utilizing cutting-edge full-wave field-solver software: Momentum (MOM) [1], EMpro (FDTD) [2] along with a hardware boost with heterogeneous massive CPU/GPU parallel processing (CUDA) technology [3], we can move the EMI teams from the back-end black-magic to a successful cost-effective front-end design. This paper presents an innovative process (VirtualEMI lab) for pre- and post-tape-out providing the designers with an early stage EMI-suppression matrix (on-chip and onboard enablers) to find the optimum trade-off between performance and cost.
\end{abstract}

\section{What is Virtual EMI Lab}

Most of EMI problems are large scale and EMI simulation requires extreme long time and excessive memory, which often makes simulation formidable for EMI engineer. Currently, EMI debug/analysis is done in the costly EMI chambers which can cost thousands of dollars and critical delays in time-to-market. However, with recent advances in simulation tools adopting CPU/GPU parallel processing technology[3], complex EMI problems are now feasible to solve within reasonable amount of time (for ex: 10-hrs with GPU instead of 8-days on the CPU).

This paper develops a pre/post tape-out process named "Virtual EMI lab" to use the EMI simulation to derive layout guidelines and to aid EMI analysis (Fig. 1). Virtual EMI lab was used to analyze various EMI problems, such as the grounding-effectiveness of the connector-to-PCB, reducing common-mode noise injected into the cables. It was also used to investigate SSO memory emission within couple of hours on the GPU to provide the best on-board decoupling-scheme for minimum radiation of the PCB antenna [5].

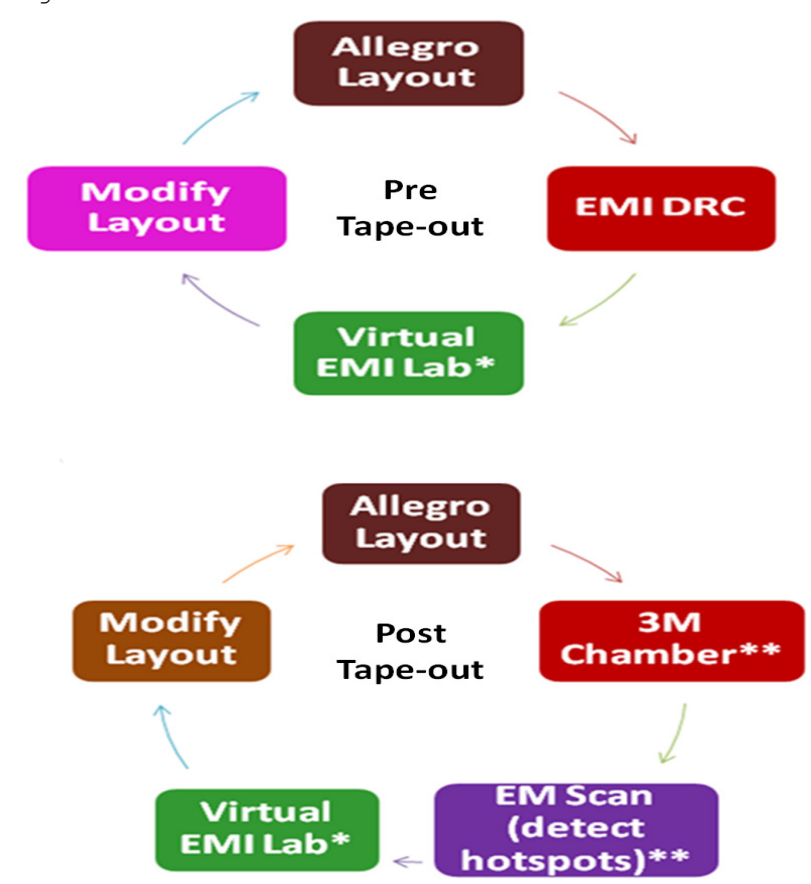

Fig. 1: Proposed Pre (top) \& Post (bottom) Tape-out Virtual-EMI lab process to develop EMI guidelines early in the design phase and efficient root-cause \& debug with mix of simulation \& measurements

\section{Momentum Providing Design-Guidelines for Memory Channel routing on Low-Cost Four-Layer PCBs}

EMI engineers want to understand the sources of EMI early on to assure best EMI-performance without the need to revert to costly EMI-suppressors such as shielded connectors, chokes, absorbers, on-board decoupling, or specially-designed mechanical enclosures. Method-of-Moments (MoM) is particularly well suited to find EMI problems in high speed boards and packages. In MoM, all metal layers are discretized with electric-surface current elements, while the voids are sampled with magnetic current elements. Special attention to the number of elements is required to assure convergence of the numerical computation of the surface current elements.

\footnotetext{
* Courtesy of Dr. P. Wesling, C.P.M.T. publications V.P.
} 
The far-fields are computed from the surface current elements, therefore, the antenna-gain is also computed.

First, a correlation of the MoM S-parameter simulation is performed: comparing to VNA measurements up to $20 \mathrm{GHz}$ on the data-signals as shown in Fig. 2), which demonstrates good accuracy of the tool. The test structure is a real 8-layer memory board and adjacent traces are terminated in 50-ohms in order to get a clean measurement. The same setup is replicated in the simulation for comparison.

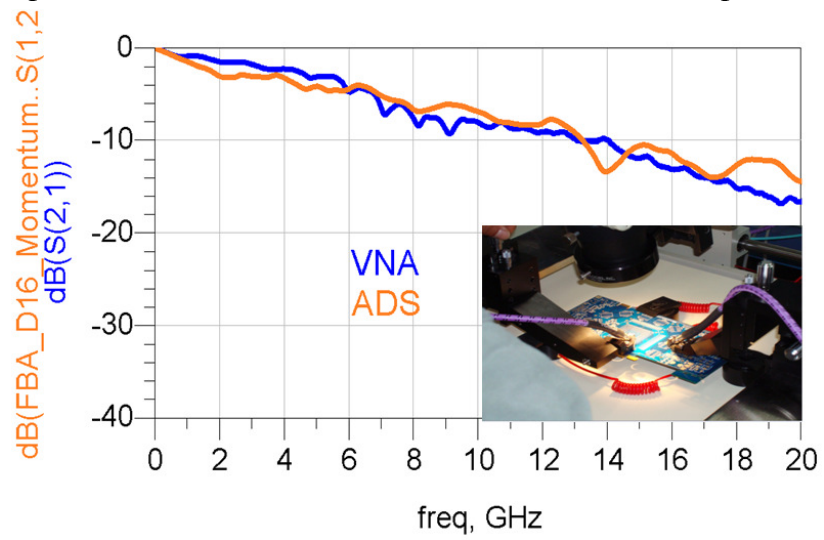

Fig. 2: S-parameter Insertion-Loss Correlation of VNA measurements with Momentum Simulations on a data-signal for the GPU-card up to $20 \mathrm{GHz}$.

Next, a 4 layer board file was imported into Momentum to investigate emission problem at 1.25 of the memory clock frequency (1.623GHz) (Fig. 3). On this board, some address/command nets are routed on bottom-layer, and they are referencing to VDDQ power plane. It was done due to lack of real-estate on a 4-layer low-cost board. The Momentum simulation shows that the Address/Command bus routed on the bottom layer, for such low-cost four-layer PCB, referencing a small $\lambda / 8$ power-plane-patch is the root-cause of the EMI emission at $1.623 \mathrm{GHz}$ (Fig. 4) and contributes $\sim 3 \mathrm{~dB}$ of Antenna-Gain(Fig. 5). The PCB is working as a nonintentional radiator. The Address/Command irregular routing on the bottom-layer was done owing to the lack of the available real-estate on a low-cost 4-layer board.

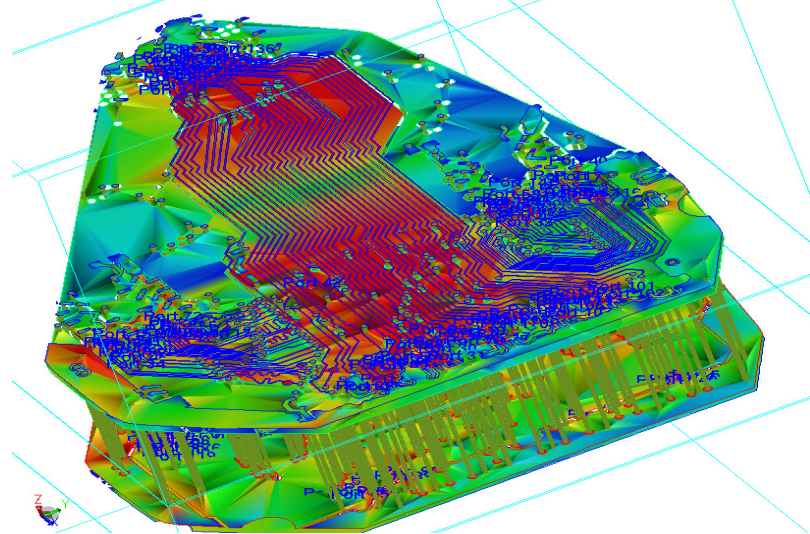

Fig. 3: Four-layer PCB simulation of memory traces of several byte-lanes, showing current-density plot.

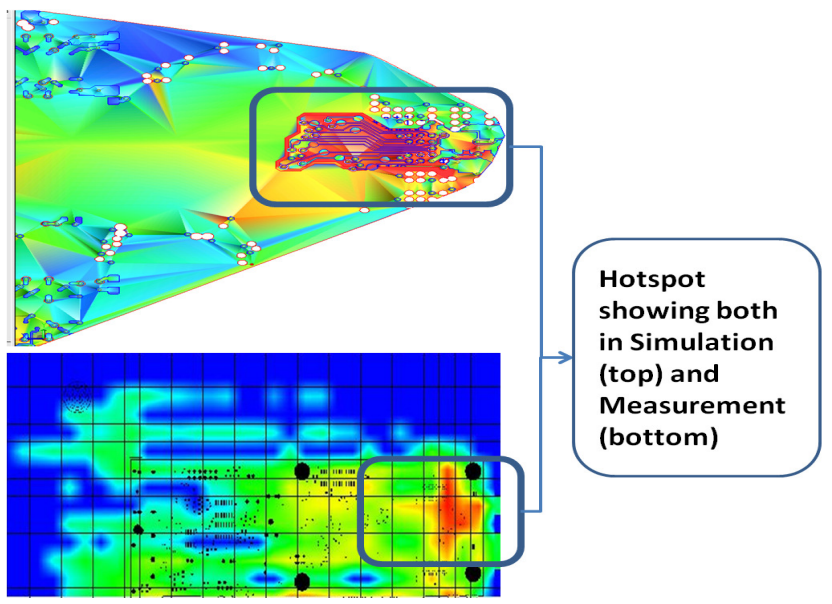

Fig. 4: Method-of-Moments (Momentum) Simulations (top) showing hot-spot of surface current on the $\lambda / 8$ powerplane patch correlating with near-field scanning hot-spot location(bottom)

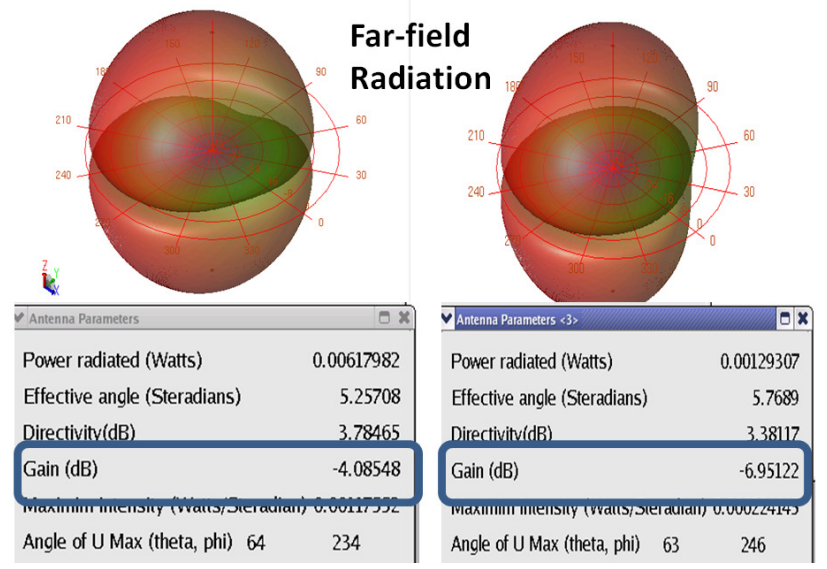

Fig. 5: Antenna-Gain with all Address/Command signals turned-on (left) is $-4 \mathrm{~dB}$ and with Address/Command signals routed on-bottom of the PCB turned-off (right) is $-7 \mathrm{~dB}$.

Using the Antenna-gain of the PCB as an optimization parameter, the best routing guidelines were developed. The MoM simulation shows that changing the shape of the $\lambda / 8$ power-plane-patch into a $\mathrm{T}$-shaped power-plane extending from the GPU to the memory devices without incorporating major return-path discontinuity (with good number of Powerstitching vias and at practical proximity of signal transitions) provided much better EMI performance (Fig. 6). 3M EMI chamber lab measurements confirmed that the new designguideline of address/command bus (T-shape versus $\lambda / 8$ patch) reduced the EMI emission by $6 \mathrm{~dB}$ (complete system with cables) correlating with the trend seen by the MoM simulations of the bare-PCB ( $3 \mathrm{~dB}$ gain).

The simulation accurately predicted that some address signals crossing the slot between the power- and groundplanes are causing large spread of hot-spots on the groundplane that inject common-mode noise into the cables connected to the GPU board; thus becoming an additional source of EMI-radiation besides the non-intentional PCB antenna. 

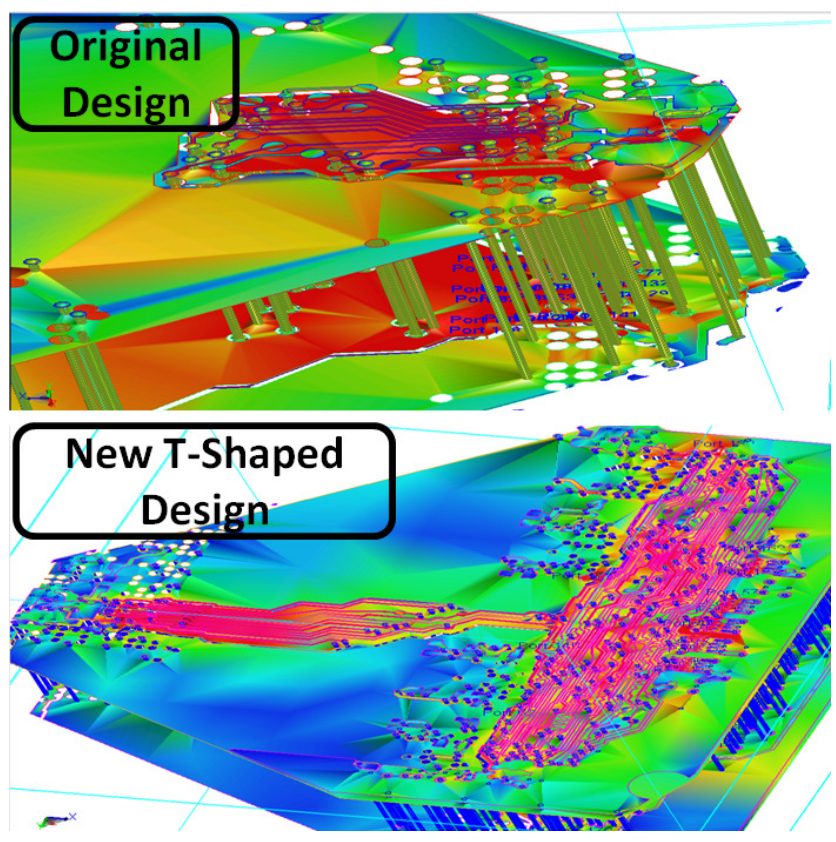

Fig. 6: Original design showing strong hot-spot around $\lambda / 8$ reference plane (top). Modified design with T-shape reference plane that damps the EMI emission by $6 \mathrm{~dB}$ (3M-chamber)

\section{Fast Root-Cause/Analysis/Debug of EMI- Emission Problems using MOM and FDTD}

Virtual-EMI lab can better support EMI engineers during post-tape-out EMI-certification phase and reduce the trial-and error EMI-suppression matrix toward faster-product-to-market (Fig. 1). The paper shows also a second example of an EMI radiation problem by TMDS signals at $770 \mathrm{MHz}$ caused by a low-cost four-layer PCB routing TMDS on the bottom layer and coupling the GPU card to the neighbor Ethernet-card causing the Ethernet-cable to radiate. Two EMI-suppressors were proposed: using a mechanical metal-shield or R4N EMIsuppressor material (Fig. 7). The MoM simulations of the bare-PCB show that the R4N suppression material is more effective in suppressing the EMI emission and actually it shows that the metal-shield is making the EMI radiation worse (Fig. 8). Near-field scanning and 10m EMI lab scan-data (Fig. 9) correlate with the predicted MOM simulations and therefore, the paper proposes a post-tape-out EMI process to enable fast debug and analysis of EMI radiation problems to prevent impact on product-performance and time-to-market. Currently the post-tape-out root-cause/debug/analysis is done in the costly EMI chambers which can cost thousands of dollars and critical delays in time-to-market) while the VirtualEMI-lab provides cost-effective and efficient process for debugging EMI problems. Emulating the EMI chamber was a dream in the near-past but now with accurate software-tools such as EMpro (FDTD) and massive parallel processing with GPU-compute capability, complex EMI problems such as $\mathrm{PCB}+$ Connector are now feasible to solve within reasonable amount of time (10-hrs on the GPU instead of 8-days on the CPU)(Fig.10)
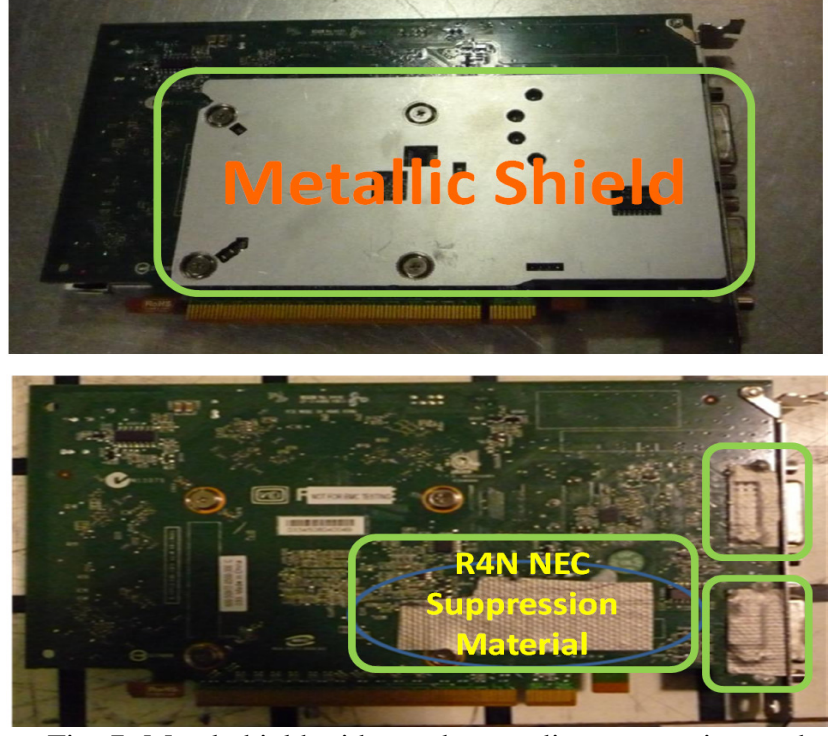

Fig. 7: Metal-shield with good grounding connection to the PCB and bracket of the GPU-card (top) and R4N NEC Suppression material placed on the TMDS signal routing as well as on the two connectors (bottom).

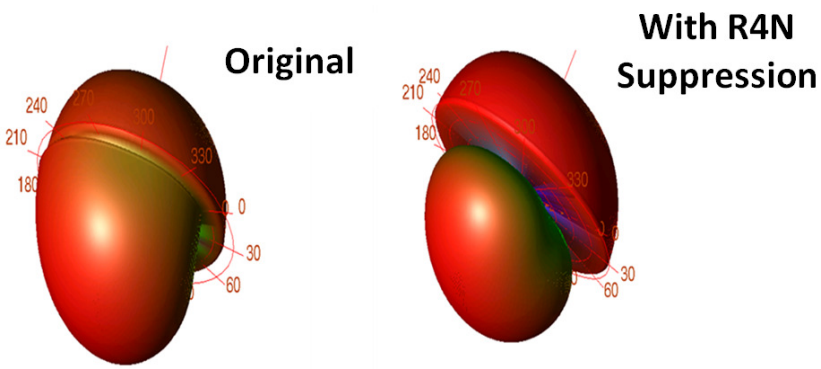

Fig. 8 EMI radiation-simulations (MoM) of the original PCB(left) and damped EMI radiation using the suppression material

(right).
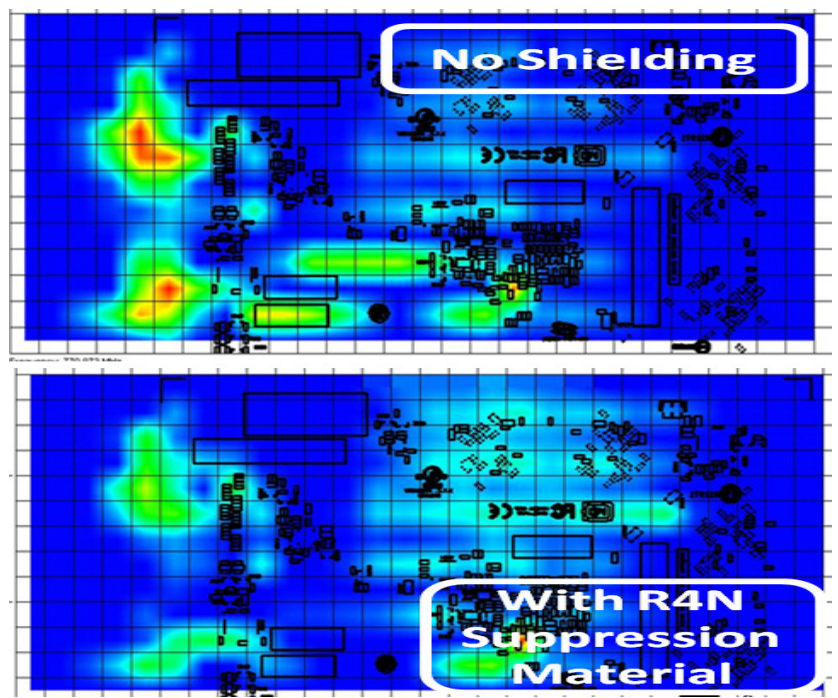

Fig. 9: near-field scanning that shows hot-spots on the original-PCB (Top) has been damping considerably by the R4N suppression material (Bottom). 
The major benefit is to analyze the grounding-effectiveness of the connector-to-PCB and reduce the common-mode noise injected into the cables to suppress the global EMI emission. Further complex problems such as SSO memory emission can be also solved accurately within couple of hours on the GPU to provide the best on-board decoupling-scheme for minimum radiation of the PCB antenna [4]
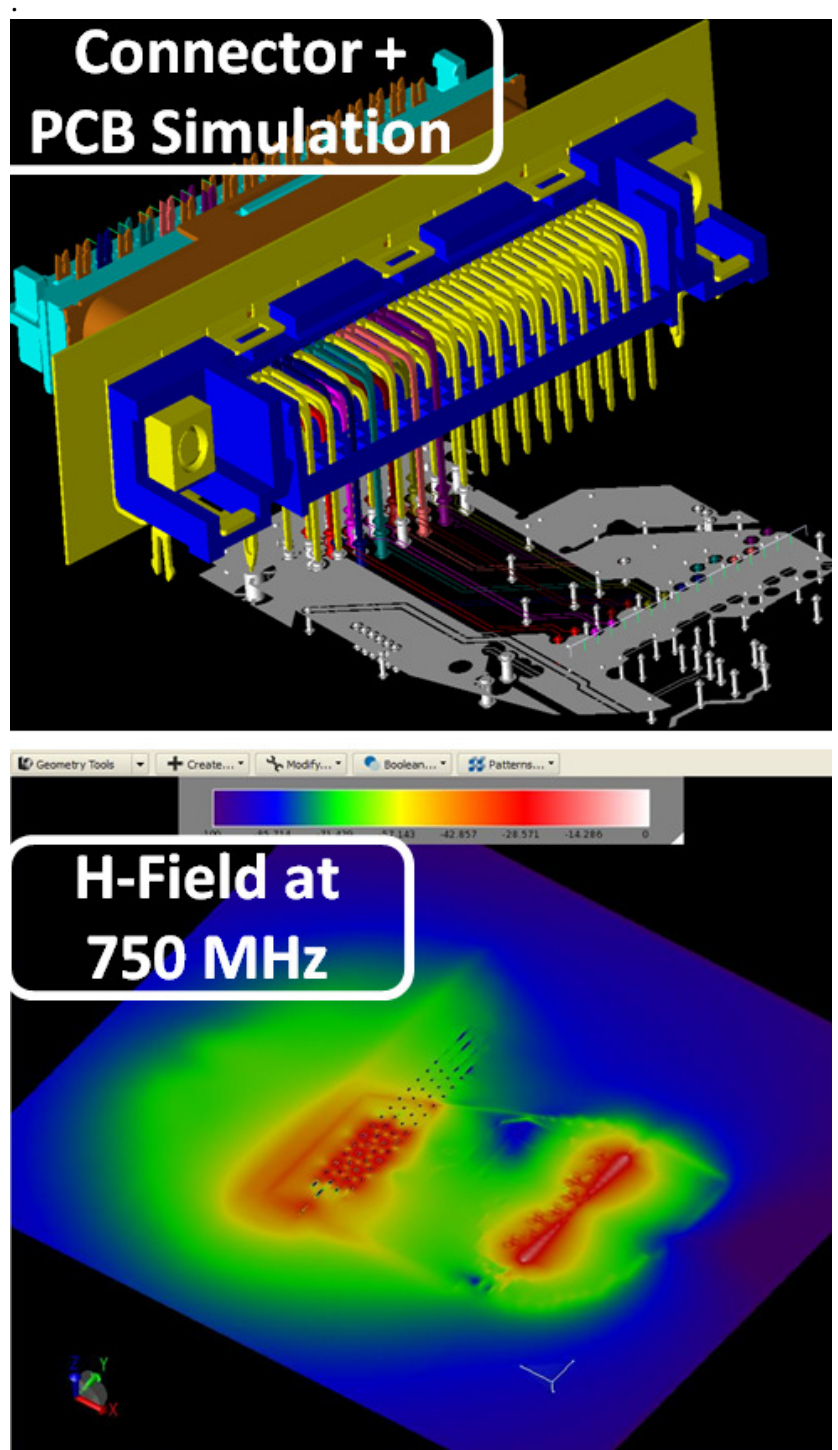

Fig. 10: showing the magnetic-fields FDTD simulations of a PCB+connector of TMDS signals at $770 \mathrm{MHz}$ that runs in 10-hrs using the GPU acceleration instead of 8-days on the CPU.

\section{Conclusions}

The paper presents a new process of "Virtual-EMI lab" for pre- and post-tape-out EMI analysis that maximizes the productivity of EMI engineers while minimizing the projectoperational expenses and keeping pace with the aggressive low-cost and time to market product requirements.

\section{Acknowledgments}

Special thanks to Giga-Test labs for S-parameter VNA measurements.

\section{References}

[1] Harrington RF, Field Computation by Moment Methods, The MacMillan Co., New York, 1968 .

[2] Dennis S, Electromagnetic Simulation Using the FDTD Method, Wiley-IEEE Press, July 2000.

[3] Kirk David, Programming Massively Parallel Processors: A Hands-on Approach.

[4] Wasserman, Y, "Integrated Single-Wafer RP Solutions for 0.25-micron Technologies," IEEE Trans-CPMT-A, Vol. 17, No. 3 (1995), pp. 346-351. [A reference to a journal article ...] 